\title{
Seguridad del paciente
}

\author{
A. MUIÑO MÍGUEZ, A. B. JIMÉNEZ MUÑOZ' B. PINILLA LLORENTE, \\ M. E. DURÁN GARCÍA ${ }^{2}$, F. J. CABRERA AGUILAR, M. P. RODRÍGUEZ PÉREZ ${ }^{1}$ \\ Medicina Interna 2A. ${ }^{1}$ Medicina Preventiva y Gestión de Calidad. ${ }^{2}$ Farmacia. Hospital \\ Universitario Gregorio Marañón. Madrid
}

PATIENT SAFETY

\section{RESUMEN}

La seguridad del paciente es un factor esencial de la calidad asistencial y desde la publicación del informe "Errar es humano" es objeto de atención general. Las estrategias de mejora han estimulado el desarrollo de modelos que permiten un mejor conocimiento de los efectos adversos ligados a la asistencia sanitaria. Los sistemas de comunicación de efectos adversos generan información que permitirá adoptar medidas que incrementen la calidad asistencial. Los efectos adversos más comunes son los relacionados con el uso de medicamentos y con frecuencia son evitables. Para disminuirlos, detectarlos y mitigarlos cuando se producen, se pueden emplear estrategias dirigidas a reducir la complejidad, optimizar la información y la automatización de procesos. Aunque el progreso sea lento los cambios se están acelerando especialmente en la implantación de sistemas de prescripción electrónica y difusión de prácticas seguras.

PALABRAS CLAVE: Seguridad del paciente. Efectos adversos. Calidad asistencial.

\begin{abstract}
Ensuring patient safety is essential for better heath care. Safety have gripped public attention ever since the release of the report "To Err is Human". To find strategies of promotion of patient safety has stimulated models that improve knowledge of adverse events. Adverse drug events are the most common cause of injury to hospitalized patients and are often preventable. Many tactics are available to make system changes to reduce errors and adverse events; they fall into five categories: Reduce complexity, optimise information processing, automate wisely, use constraints, and mitigate the unwanted side effects of change. These tactics can be deployed to support any of the three strategic components of error prevention, detection, and mitigation. Although progress has been slow, the pace of change is likely to accelerate, particularly in implementation of electronic health records and diffusion of safe practices.
\end{abstract}

KEY WORDS: Patient safety. Adverse events. Quality of Care.

Muiño Míguez A, Jiménez Muñoz AB, Pinilla Llorente B, Durán García ME, Cabrera Aguilar FJ, Rodríguez Pérez MP. Seguridad del paciente. An Med Interna (Madrid) 2007; 24: 602-606.

\section{INTRODUCCIÓN}

La medicina ha pasado de ser simple, poco efectiva y relativamente segura a convertirse en compleja, efectiva pero potencialmente peligrosa (1).

En efecto la atención sanitaria es cada vez más compleja: los procesos asistenciales suponen una combinación de actuaciones, tecnologías e interacciones humanas de un gran número de profesionales. Esto se asocia a un riesgo creciente de efectos adversos y perjuicio involuntario para el paciente.

Los efectos adversos son los daños, o lesiones que acontecen durante el proceso asistencial y, al no estar directamente producidos por la enfermedad en sí, se pueden considerar motivados por el propio sistema sanitario, ya sea por acción u omisión. Un incidente es una acción o conjunto de acciones por comisión u omisión que podría haber dañado al paciente, pero que este daño no ha llegado a producirse, es decir, que difiere de un efecto adverso "sólo" en el resultado para el paciente. La diferencia con las complicaciones es clara, ya que éstas últimas suponen alteraciones del proceso natural de la enfermedad, derivada de la misma y no provocada por la actuación médica.

Uno de los principios clásicos de la medicina es el hipocrático "primum non nocere", lo que indica que siempre se ha reconocido la posibilidad de que la actuación médica produzca consecuencias indeseables. Sin embargo la información sobre errores médicos y EA es muy reciente ya que los primeros estudios datan de los años 60 y no es hasta la década de los 90 cuando se comenzó a disponer de evidencia científica. Pero es en el año 99 cuando el impacto entre los profesionales, autoridades y público general del informe To Err is Human: Building a Safer Health System del Instituto de Medicina de EE.UU. (2), marca un antes y un después en la concienciación sobre este tema y ha hecho que la investigación sobre seguridad del

Trabajo aceptado: 18 de julio de 2007

Correspondencia: Antonio Muiño Míguez. Hospital Gregorio Marañón. Medicina Interna 2A. C/ Doctor Esquerdo, 46. 28007 Madrid. e-mail: antonio.muino@madrid.org 
paciente y las iniciativas para actuar sobre los riesgos evitables sean una prioridad para todos las autoridades sanitarias. Este documento refleja la magnitud del problema de seguridad de la asistencia y la enorme repercusión sanitaria y económica que implica: Estima por extrapolaciones basadas en la revisión de historiales médicos que cada año se producen en EE.UU. un millón de efectos adversos cuyas consecuencias serán que entre 44.000 y 98.000 pacientes mueren cada año por errores médicos, cifras que superan la mortalidad de los accidentes de automóvil (43.000 víctimas), cáncer de mama (42.000) o el SIDA (16.000). Aunque algunos pueden considerar estas cifras exageradas (3), disponemos de numerosos estudios que en cualquier caso reconocen la importancia del problema, ofreciendo cifras variables según el tipo de evaluación realizada y el concepto más o menos amplio que se utilice: Zhan y Miller (4) examinaron la incidencia de efectos adversos en 18 hospitales, concluyendo que los efectos adversos pueden ser responsables en EE.UU. de 2.4 millones de días de estancia hospitalaria y 32.000 muertes. Davis et al (5) encontraron $12,9 \%$ de efectos adversos durante las hospitalizaciones en Nueva Zelanda. El Departamento de Salud del Reino Unido, en su informe del año 2000, An organization with a memory (6), estimó que se producen eventos adversos en cerca del $10 \%$ de las hospitalizaciones, es decir 850.000 eventos al año. En Australia, la tasa de eventos adversos descrita fue del $16,6 \%$ entre los pacientes ingresados (7). Por tanto las frecuencias de efectos adversos en los diferentes estudios se sitúan entorno al $10 \%$ de los pacientes que acuden a un hospital, con un rango que varía desde el 4 al $17 \%$.

Las consecuencias y costes de estos EA han sido estimados por las diferentes instituciones sanitarias. Y así en el Reino Unido, se ha calculado que el exceso de estancias hospitalarias origina un gasto de 2.000 millones de libras al año, a lo que hay que sumar los 400 millones/año por indemnizaciones, los 2.400 millones/año por reclamaciones judiciales y los 1000 millones /año que cuestan las infecciones nosocomiales. En EE. UU se calcula que los errores prevenibles son responsables del 12 al $15 \%$ de los costes hospitalarios (8) y que se origina un exceso de estancia media en EA prevenibles de 4, 5 días y 4700 dólares (9)

A estos costes tangibles habría que añadir la erosión de la confianza, de la seguridad y de la satisfacción del público y de los proveedores de atención sanitaria.

\section{GESTIÓN DE RIESGOS}

Mejorar la seguridad de los pacientes, como se ha comentado, se ha convertido en un objetivo prioritario en las políticas de calidad de los sistemas sanitarios y se han adoptado estrategias por diversos organismos internacionales (UE, OMS, OCDE, etc.) para abordar la ocurrencia de eventos adversos relacionados con la asistencia sanitaria.

La gestión de riesgos es una nueva disciplina que tiene como objetivo el estudio de los efectos adversos derivados de la asistencia mediante su detección y análisis, con el objetivo final de diseñar estrategias para su prevención, generando una cultura de preocupación por la seguridad, ya que ésta es probablemente la dimensión más importante de la calidad asistencial. Toda actividad humana conlleva un margen de error, controlar y minimizar ese posible daño es la gestión del riesgo. $\mathrm{Su}$ práctica debe ser en equipos multidisciplinarios con abordajes epidemiológicos, clínicos y organizacionales, y debe estar integrada en programas de calidad. Las Unidades de Gestión de Riesgos pueden tener distintos objetivos según el medio en el que desarrollen su labor, pero su denominador común es buscar una mejora de la calidad asistencial desarrollando sistemas de notificación de incidentes, investigación de los mismos, asesoramiento en las reclamaciones, difusión de la cultura de la seguridad, evaluación de las situaciones de riesgo y propuesta de medidas correctoras, que conlleven un seguimiento y evaluación de dichas actuaciones de mejora.

En resumen la gestión de riesgos se realiza en tres fases:

- Identificación

-Evaluación-análisis: de la frecuencia, el coste y la gravedad del riesgo

- Tratamiento: que conlleva el conjunto de actuaciones para prevenir el riesgo eliminándolo, y si no es posible reducirlo.

\section{DETECCIÓN DE RIESGOS}

Los errores pueden clasificarse según su evitabilidad, las actividades de prevención van siempre dirigidas a minimizar la exposición a los riesgos en el caso de los riesgos inevitables y a la prevención en el caso de los evitables. Por otro lado la mayoría de los errores evitables en organizaciones complejas están relacionados más con el sistema que con fallos individuales.

La creación de sistemas de registro y notificación de problemas de seguridad constituyen una estrategia clave para aprender de los errores y evitar su recurrencia en el contexto de la mejora continua $(2,10)$.

No hay unanimidad sobre cual es el sistema de notificación ideal. Pueden cumplir dos funciones: se pueden orientar hacia garantizar la responsabilidad social (de tal forma que los proveedores rindan cuentas sobre la seguridad de su práctica) o, de forma alternativa o complementaria, para que los proveedores proporcionen información útil sobre la mejora de la seguridad.

Los sistemas que se enfocan hacia la mejora de la seguridad son los sistemas de carácter voluntario. Se centran en los incidentes (donde no ha habido daño) o en errores que han producido daño mínimo. Su objetivo principal es identificar áreas o elementos vulnerables del sistema antes de que se produzca el daño en los pacientes y formar a los profesionales sobre lo aprendido con el análisis de múltiples casos $(11,12)$.

Los profesionales sanitarios implicados en la práctica diaria son los idóneos para la detección de los problemas, pero deben contar con el apoyo de expertos en la metodología a aplicar en su análisis que además difundan los conocimientos necesarios para poner en marcha los programas.

El temor a la responsabilidad individual puede ser un obstáculo importante a la declaración efectiva de los efectos adversos y por tanto llevar a un desconocimiento de la verdadera magnitud de los problemas por la propia organización, impidiendo así adoptar medidas correctoras. Deben tomarse medidas, incluidas las legislativas, que eviten que ésto suceda. En este sentido se están dando los primeros pasos en varios países para modificar la legislación.

Los sistemas de registro y notificación no pretenden ser una estimación de la frecuencia de efectos adversos e incidentes en el sector sanitario, sino una forma de obtener información sobre la cascada de acontecimientos que llevan a su producción. 
Los efectos adversos son el vértice de una pirámide cuya base son los incidentes compartiendo unos y otros las mismas causas, por lo que el aprendizaje del análisis de las causas de los incidentes es también útil para la prevención de los efectos adversos (13).

Para aumentar la confianza de los profesionales hacia los sistemas de notificación y potenciar su uso deben garantizar la protección de los datos y que no se revelan públicamente, que nunca se deriven represalias, orientado más a sistemas que a la práctica individual y que se perciba su utilidad (14).

\section{EPIDEMIOLOGÍA DE LOS RIESGOS}

Los estudios sobre la epidemiología de los riesgos de la atención sanitaria coinciden en identificar las complicaciones relacionados con los medicamentos como la causa más frecuente de efectos adversos seguidos de los derivados de intervenciones quirúrgicas y de las infecciones nosocomiales (15).

En relación con los riesgos derivados del uso de los medicamentos, aunque la mayoría de los errores son poco peligrosos, o son interceptados, otros llegan a producir daño, Bates et al identificaron eventos adversos relacionados con los fármacos en el 6,5\% de los pacientes ingresados en un Hospital Universitario de Boston (16) y en el 19\% del total de acontecimientos adversos registrados en el Harvard Medical Practice Study (17) en 51 hospitales de Nueva York; el 4\% de los registrados en el estudio de Neale et al. (18) en dos hospitales de Londres, y el $24 \%$ en el Canadian Adverse Events Study (19). Entre el 28 y el $56 \%$ de los errores de medicación se consideran evitables.

Esto justifica el gran interés dirigido a estudiar las fases en las que se pueden producir los errores, (prescripción, trascripción, dispensación, administración), y las medidas dirigidas a evitarlos.

El segundo lugar por la magnitud del problema, están las complicaciones perioperatorias. Un estudio realizado sobre 30.195 altas en Nueva York (17), indicaba que sobre el total de los efectos adversos detectados el 13,3\% se debía a infección de la herida quirúrgica (y de éstos un 12,5\% eran debidos a negligencias), un $12,9 \%$ de los efectos adversos producidos se debían a complicaciones en la técnica quirúrgica y un $10,6 \%$ eran complicaciones tardías de la cirugía.

En lo que se refiere a la infección nosocomial, en España se cuenta con datos desde el año 1990, en el que se realizó por primera vez el Estudio de prevalencia de Infecciones nosocomiales (EPINE). Este estudio en el año 2005 arroja una tasa de prevalencia de infección nosocomial de $6,9 \%$, frente a un $8,5 \%$ del año 90, apreciándose una tendencia descendente estadísticamente significativa (21), posiblemente en relación a actuaciones preventivas como el uso de sondajes urinarios cerrados o la introducción en los hospitales de las soluciones alcohólicas para el lavado de manos.

Por otro lado en cuanto a la distribución de riesgos según especialidades médicas, diversos estudios ponen de manifiesto que son más frecuentes en servicios quirúrgicos. Un estudio realizado sobre 15.000 altas no psiquiátricas en EE.UU. obtuvo que un $46,1 \%$ del total de los riesgos detectados se produjo en cirugía, siendo el $22,3 \%$ errores evitables. Un $23,2 \%$ en medicina interna, en donde los errores considerados evitables eran el $44,9 \%(22)$.

\section{ANÁLISIS DE RIESGOS}

Dos son las herramientas utilizadas para el análisis y evaluación de riesgos sanitarios: El análisis modal de fallos y efectos, también llamado por su acrónimo AMFE y el Análisis causa- Raíz.

El AMFE es una técnica creada para el proyecto APOLO en la década de los 60, que se ha ido extendiendo a la aeronáutica, la energía nuclear y al resto de las industrias y empresas de servicios. Se trata de una técnica analítica que identifica los posibles fallos de cada actividad de un proceso, evalúa los efectos que pueden ejercer los fallos sobre el cliente-paciente, identifica posibles causas y determina las medidas de control para la prevención o detección de las condiciones del fallo. Sus características principales son:

- Tener un carácter preventivo: se anticipa a la ocurrencia del fallo en los productos, servicios o en los procesos

- Ser sistemático: Es un enfoque estructurado que asegura prácticamente, que todas las posibilidades de fallo han sido contempladas

- Sirve de guía en la priorización de las acciones necesarias para anticiparse a los problemas

-Es un sistema participativo: se trata de un trabajo en equipo con puesta en común de todas las áreas afectadas

- Aplicación a nuevos productos, servicio o para los ya existentes

El Análisis de causas-raíz , se utiliza cuando ya se ha identificado una situación de partida para el análisis (un incidente). Se trata, por lo tanto de una herramienta de prevención secundaria en la que con una serie de preguntas estructuradas se intenta descubrir errores raíces que originan el efecto adverso. Para ello se realiza una revisión exhaustiva de las personas, el equipo, los procedimientos... Al final del proceso se definen las causas próximas, entendiendo éstas por aquellos actos u omisiones que producen una consecuencia directamente y que al tratarla no evitarían la reaparición del fallo y las causas raíz u origen que son las fundamentales para la aparición del fallo o la ineficiencia. Si actuamos sobre ella se evita la aparición del fallo.

\section{ESTRATEGIAS DE ACTUACIÓN-TRATAMIENTO}

Los sistemas deben diseñarse para prevenir los errores, facilitar su detección y permitir que si se producen provoquen el menor daño posible.

Las estrategias para mejorar los procesos y reducir los errores pueden clasificarse en cuatro grupos (20):

1. Reducir la complejidad. La complejidad causa errores y aunque algunos procedimientos diagnósticos y tratamientos, así como casos individuales son complejos en si mismos, en otras ocasiones es posible la simplificación. Diferencias en las dosis o formas de administración de medicamentos, diferencias en la organización de las unidades de un mismo hospital, dependen en muchas ocasiones más de preferencias personales que de evidencias científicas. Por otra parte la organización del hospital en "compartimentos" poco cohesionados provoca que la asistencia no sea lo integral y estructurada que desearíamos. Este tipo de organización favorece la aparición de efectos adversos (23).

2. Optimizar la información. Evitar en lo posible que la asistencia dependa de la memoria de los profesionales y utilizar protocolos, vías clínicas, checklists, etc. 
3. Automatización. La utilización de tecnologías como la prescripción electrónica asistida, automatizar la preparación de medicación, dispensadores de medicación, pueden ser de utilidad. No obstante siempre debe considerarse la automatización de procesos y la incorporación de nuevas tecnologías no como un fin en sí mismo, el objetivo es la mejora del proceso, usar la tecnología para facilitar el trabajo del personal no para sustituirlo.

4. Implantar las innovaciones con las mayores precauciones pues son períodos en los que los errores pueden producirse con más facilidad.

Las recomendaciones siguientes se desprenden del análisis de los informes internacionales:

- Establecer un enfoque consistente para conseguir liderazgo, investigación, herramientas y protocolos para potenciar el conocimiento sobre la seguridad de los pacientes.

- Identificar los problemas de seguridad y aprender de ellos mediante sistemas de registro y notificación de carácter obligatorio o voluntario

- Formular estándares de mejora de la seguridad por parte de gobiernos, organizaciones, sociedades científicas y profesionales.

- Implementar prácticas seguras en los niveles de la prestación asistencial.

Para desarrollar estas recomendaciones se pueden desplegar diferentes estrategias concretas como por ejemplo la formación de profesionales, la detección precoz y sistemática de riesgos en las áreas de atención al paciente, el análisis de los casos en los que se ha producido un evento adverso, la comunicación con los pacientes para que mejoren su conocimiento sobre su propia seguridad y el registro y notificación de eventos adversos, entre otras.

Entre las recomendaciones aceptadas como eficaces para disminuir estos y otros riesgos estarían, junto al rediseño de procesos: Garantizar la identificación inequívoca de los pacientes. Impulsar la historia clínica electrónica. Implantar la prescripción electrónica asistida. Estrategias para evitar las discrepancias no intencionadas con la medicación habitual al inicio de la hospitalización (24-28).

Se considera que los sistemas informáticos de prescripción médica son una de las principales medidas para mejorar la seguridad del paciente. Permite eliminar muchos problemas asociados con la prescripción manual como órdenes ilegibles, dosis inadecuadas, o prescripciones incompletas, obteniéndose disminuciones en los errores médicos del 55 al $80 \%$. Sin embargo no se ha demostrado que estos sistemas disminuyan la mortalidad, aunque parece evidente la reducción de errores menos graves. Obviamente no todos los sistemas son iguales y muchos incorporan sistemas de ayuda que incrementan su eficacia (29).

\section{SITUACIÓN EN ESPAÑA}

En nuestro país también se han dado pasos significativos. El Estudio Nacional sobre los Efectos Adversos ligados a la Hospitalización (ENEAS 2005) (30) supone un importante punto de partida en el conocimiento de nuestra realidad. Este estudio tenía como objetivos principales:

- Determinar la incidencia de Efectos Adversos (EAs) y de pacientes con EA en los hospitales de España.

-Establecer la proporción de EAs que se producen en el período de prehospitalización.
- Describir las causas inmediatas del EA.

- Definir los EAs evitables.

- Conocer el impacto de los EAs en términos de incapacidad, éxitus y/o prolongación de la estancia hospitalaria.

Entre sus resultados destacan:

Se detectaron 1.063 pacientes con EA durante la hospitalización, siendo la incidencia de pacientes con EAs relacionados con la asistencia sanitaria de 9,3\% (525/5.624); IC95\%: $8,6-10,1 \%$. La incidencia de pacientes con EAs relacionados directamente con la asistencia hospitalaria (excluidos los de atención primaria, consultas externas y ocasionados en otro hospital) fue de 8,4\% (473/5.624); IC95\%: 7,7-9,1\%. El $17,7 \%$ de los pacientes con EA acumuló más de un EA. Del total de los 473 pacientes con EAs relacionados con la hospitalización, en $105(22,2 \%)$ el EA fue la causa del ingreso hospitalario (reingreso). El 42,8\% de los EAs se consideró evitable, en función de los criterios prefijados. La gravedad de los EAs también se asoció a su evitabilidad, de tal forma que los EAs leves eran evitables en un 43,8, los moderados lo eran en un 42 y los graves en un $41,9 \%$ aunque estas diferencias no alcanzaron significación estadística.

El Plan de Calidad para el Sistema Nacional de Salud establece como una de sus estrategias prioritarias: Mejorar la seguridad de los pacientes atendidos en los centros sanitarios del SNS, estableciendo entre otras las siguientes líneas de actuación:

- Promover y desarrollar el conocimiento y la cultura de seguridad del paciente entre los profesionales en cualquier nivel de atención sanitaria.

- Diseñar y establecer sistemas para la comunicación de los incidentes relacionados con la seguridad del paciente.

-Difundir los resultados del Estudio Nacional de Efectos Adversos.

- Realizar un estudio sobre la percepción de los profesionales del Sistema Nacional de Salud hacia la seguridad de pacientes.

- Establecer una línea prioritaria de investigación básica y aplicada en seguridad de Pacientes a través del Fondo de Investigaciones Sanitarias.

- Suscribir la declaración conjunta con la OMS para impulsar la Alianza Mundial sobre Seguridad de los Pacientes.

- Diseñar y ofertar a los profesionales, herramientas de gestión clínica y seguridad.

- Realizar una campaña de comunicación sobre seguridad de pacientes.

- Elaborar una propuesta de medidas de mejora en materia de efectos adversos

- Diseñar y establecer sistemas para la comunicación de los incidentes relacionados con la seguridad del paciente.

- Realizar un análisis de los sistemas de notificación de eventos adversos existentes y establecer un conjunto básico de indicadores mínimos en seguridad de pacientes.

- Diseñar un sistema de notificación de efectos adversos a nivel del Sistema Nacional de Salud y en las diferentes Comunidades Autónomas.

- Prevenir úlceras por presión en pacientes en riesgo.

- Prevenir el Trombo-embolismo Pulmonar (TEP)/ Trombosis Venosa Profunda (TVP) en pacientes sometidos a cirugía.

- Prevenir la infección nosocomial y las infecciones quirúrgicas.

- Prevenir los errores debidos a medicación. 
- Asegurar la implantación y correcta aplicación del consentimiento informado así como el cumplimiento de las últimas voluntades de los pacientes.

Después de más de seis años de To err is human el progreso no ha sido todo el deseado. El compromiso con la seguridad requiere la adopción de objetivos ambiciosos y

\section{TABLA I}

\section{SEGURIDAD DEL PACIENTE EN SIETE PASOS (SEGÚN}

SISTEMA NACIONAL DE LA SALUD DEL REINO UNIDO)

1. Construir una cultura de seguridad

2. Liderazgo del equipo de personas

3. Integrar las tareas de gestión de riesgos

4. Promover que se informe

5. Involucrarse y comunicarse con pacientes y público

6. Aprender y compartir lecciones de seguridad

7. Implementar soluciones para prevenir daños explícitos. Aunque de la implantación de nuevas tecnologías con sistemas informáticos amigables implementados con protocolos adecuados y basados en la evidencia científica cabe esperar una contribución importante, es prioritario la generalización de la cultura de la seguridad (Tablas I y II) $(31,32)$.

TABLA II

EFECTIVIDAD DE ALGUNAS PRÁCTICAS SEGURAS

\begin{tabular}{ll}
\hline Intervención & Resultado \\
\hline Protocolo antibioterapia perioperatoria & Disminución infecciones 93\% \\
Prescripción asistida por ordenador & $81 \%$ reducción errores medicación \\
Farmacéuticos en equipo asistencial & $67-78 \%$ reducción errores medica- \\
ción Equipos rápida respuesta & Disminución 15\% PCR \\
Prácticas de reconciliación medicación & $90 \%$ reducción errores medicación \\
Estandarización dosis insulina & Disminución 63\% hipoglucemias \\
\hline
\end{tabular}

\section{Bibliografía}

1. Chantler $\mathrm{C}$. The role and educationa of doctors in the delivery of health care. Lancet 1999; 353: 1178-81.

2. Committee on Quality of Health Care in America, Institute of Medicine. To Err is Human: Building a Safer Health Care System. National Academy Press, Washington, D.C., 2000.

3. McDonald CJ, Weiner M, Hui SL. Deaths due medical errors are exagerated in Institute of Medicine report. JAMA 2000; 284: 93-5.

4. Zhan C, Miller MR. Excess length of stay, changes, and mortality attributable to medical injuries during hospitalisation. JAMA 2003; 290 : 1868-74.

5. Davis P, Lay-Yee R, Briant R, Ali W, Scot A, Schung S. Adverse events in New Zeland public hospitals: Occurrence and impact. NZ Med J 2002; 115: U271.

6. Department of Health. An Organization with a memory. The Stationery Office. London 2000.

7. Wilson RM, Runciman WB, Gibberd RW, Harrison BT, Newby L, Hamilton JD. The Quality in Australian Health Care Study. Med J Aust 1995; 163: 458-76.

8. Elwyn G, Corrigan JM. The patient safety story. BMJ 2005; 331: 302-4

9. Stryer D, Clancy C. Patients' safety. BMJ 2005; 330: 553-4.

10. Protti D, Catz M. The HER and Patien Safety: A paradigm shift for healthcare decision- Makers. Electronic Healthcare 2002; 1: 32-41.

11. Bates DW, Spell N, Cullen DJ, Burdick E, Laird N, Petersen LA, et al. The costs of adverse drug events in hospitalized patients. Adverse Drug Events Prevention Study Group. JAMA 1997; 277: 307-11.

12. Sistemas de registro y notificación de incidentes y eventos adversos. Ministerio de Sanidad y Consumo, 2005.

13. Michel P, Quenon JL, de Sarasqueta AM, Scemama O. Comparison of three epidemiological methods for estimating adverse events and preventability rates in acute care hospitals. BMJ 2004; 328: 199-204.

14. Bañeres J, Orrego C, Suñol R, Ureña V. Los sistemas de registro y notificación de efectos adversos y de incidentes: Una estrategia para aprender de los errores. Rev Calidad Asistencial 2005; 20: 216-22.

15. Michel P, Aranaz JM, Limón R, Requena J. Siguiendo la pista de los efectos adversos: Cómo detectarlos. Rev Calidad Asistencial 2005; 20 (4): 204-10.

16. Bates DW, Cullen DJ, Laird N, Petersen LA, Small SD, Servi D, et al. Incidence of adverse drug events and potential drug events. Implications for prevention. JAMA 1995; 274: 29-34.

17. Leape L, Brennan T, Laird N, et al. The nature of adverse events in hospitalized patients. Results of the Harvard Medical Practice Study II. N Engl J Med 1991; 324: 377-84.

18. Neale G, Woloshynowych M, Vincent C. Exploring the cause of adver-

se events in NHS hospital practice. J R Soc Med 2001; 94: 322-30.

19. Baker GR, Norton PG, Flintoft V, Blais R, Brown A, Cox J, et al. The Canadian Adverse Events study: The incidence of adverse events among hospital patients in Canada. CMAJ 2004; 170: 1678-86.

20. Nolan TW. System changes to improve patient safety. BMJ 2000; 320: 771-3.

21. Estudio de la prevalencia de las infecciones nosocomiales. Sociedad Española de Medicina Preventiva, Salud Pública e Higiene. (Consultado 1-12-2006). Disponible en: http://mpsp.org/mpsp/EPINE06/

22. Thomas EJ, Studdert DM. Burstin HR. Orav EJ; Zeena T; Williams EJ, Howard K.M, Weiler PC, Brennan TA. Incidence and Types of Adverse Events and Negligent Care in Utah and Colorado. Medical Care 2000; 38: 261-71.

23. Rosado-Bretón L, Martínez-Soriano J. Gestión de riesgos desde la visión de la gestión hospitalaria. Rev Calidad Asistencial 2005: 20: 110 4.

24. Bates DW, Cohen M, Leape LL, Overhage JM, Shabot MM, Sheridan T. Reducing the Frequency of Errors in Medicine Using Information Technology. J Am Med Inform Assoc 2001; 8: 299-308.

25. Bates DW, Cullen DJ, Laird N, et al. Incidence of adverse drug events and potential adverse drug events. Implications for prevention. ADE prevention study group. JAMA 1995; 274: 29-34.

26. Aranaz JM. Sucesos adversos relacionados con el uso del medicamento: ¿Qué podemos aprender? Med Clín (Barc) 2006; 126: 97-8.

27. Cornish PL, Knowles SR, Marchesano R, Tam V et al. Unintended Medication Discrepances at the Time of Hospital Admission. Arch Intern Med 2005; 165: 424-9.

28. Colpaert K, Claus B, Somers A, Vandewoude K, Robays H, Decruyenaere J. Impact of computerized physician order entry on medication prescription errors in the intensive care unit: A controlled cross-sectional trial. Crit Care 2006; 10: R21.

29. Wachter RM. Expected and unanticipated consequences of the quality and information technology revolutions. JAMA 2006; 295: 2780-3.

30. Estudio Nacional sobre los efectos adversos ligados a la hospitalización. ENEAS 2005. Informe. Febrero 2006. Ministerio de Sanidad y Consumo. Secretaría General de Sanidad. Dirección General de la Agencia de Calidad del Sistema Nacional de Salud.

31. Agencia Nacional para Seguridad del Paciente. Sistema Nacional de Salud del Reino Unido. La seguridad del paciente en siete pasos (Consultado 1-12-2006). Disponible en: http: //www.msc.es/ organizacion/ sns/ planCalidadSNS/ ec03_doc.htm

32. Leape L1, Berwick DM. Five years after To err Is Human. JAMA 2005, 293: 2384-90. 